\title{
Optimal Selective Maintenance Strategy for Multi-State Systems Under Imperfect Maintenance
}

\author{
Yu Liu and Hong-Zhong Huang, Member, IEEE
}

\begin{abstract}
Many systems are required to perform a series of missions with finite breaks between any two consecutive missions. In such a case, one of the most widely used maintenance policies is a selective maintenance in which a subset of feasible maintenance actions is chosen to be performed with the aim at achieving the subsequent mission success under limited maintenance resources. Traditional selective maintenance optimization reported in the literature only focuses on binary state systems. Most systems in industrial applications, however, have more than two states in the deterioration process. In this work, a selective maintenance policy for multi-state systems (MSS) consisting of binary state elements is investigated. Taking the imperfect maintenance quality into consideration, the Kijima model is reviewed, and a cost-maintenance quality relationship which considers the age reduction factor as a function in terms of maintenance cost is established. Moreover, with the assistance of the universal generating function (UGF) method, the probability of the repaired MSS successfully completing the subsequent mission is formulated. In place of enumerative methods, a genetic algorithm (GA) is employed to solve the complicated optimization problem where both multi-state systems, and imperfect maintenance models are taken into account. The effectiveness of the proposed method is demonstrated via a case study of a power station coal transportation system. Finally, a comparative analysis between the strategies with and without considering imperfect maintenance is conducted, and it is concluded that incorporating imperfect maintenance quality into selective maintenance achieves better outcomes.
\end{abstract}

Index Terms-Genetic algorithm, imperfect maintenance, maintenance cost allocation, multi-state systems, selective maintenance, universal generating function.

\section{ACRONYMS $^{1}$}

CM corrective maintenance

PM preventive maintenance

MSS multi-state system

UGF universal generating function

GA genetic algorithm

Manuscript received October 05, 2008; revised September 29, 2009; accepted November 15, 2009. Date of publication May 03, 2010; date of current version June 03,2010 . This work was partially supported by the National Natural Science Foundation of China under contract number 50775026. Associate Editor: G. Levitin.

Y. Liu and H.-Z. Huang are with the School of Mechanical, Electronic, and Industrial Engineering, University of Electronic Science and Technology of China, Chengdu, Sichuan, 611731, China (e-mail: hzhuang@uestc.edu.cn).

Color versions of one or more of the figures in this paper are available online at http://ieeexplore.ieee.org.

Digital Object Identifier 10.1109/TR.2010.2046798

${ }^{1}$ The singular and plural of an acronym are always spelled the same.

\section{NOTATION}

$M$

$n_{i}$

$\mathrm{g}_{i}$

$g_{i, j}$

$\mathbf{p}_{i}(t)$

$p_{i, j}(t)$

$G_{i}(t)$

$N_{s}$

$\mathbf{G}_{\mathbf{s}}$

$g_{j}$

$\mathbf{p}(\mathbf{t})$

$p_{j}(t)$

$G(t)$

$\phi(\cdot)$

$w_{i}$

$q_{i}$

$A_{i}(k)$

$B_{i}(k)$

$X_{i} k$

$Y_{i}(k)$

$L(k)$

$c_{i}^{0}$

$c_{i}^{r f}$

$c_{i}^{r p}$

$c_{i}(k)$

$C_{i}(k)$

$b_{i}(k)$

$\eta_{i}$
Number of $s$-independent elements in the MSS

Number of states for element $i$

Set of possible performance rates of element $i$

Performance rate of element $i$ in state $j$

Set of state probabilities for element $i$ at time $t$

Probability of element $i$ staying at state $j$ at time $t$

Random variable representing the performance rate of element $i$ at time $t$

Number of states for the MSS

Set of possible system performance rates

Performance rate of the MSS in state $j$

Set of system state probabilities at time $t$

Probability of the MSS being in state $j$ at time $t$

Random variable representing the system

performance rate at time $t$

MSS structure function

The $i$ th possible demand level in the next mission

Probability of the demand level being equal to $w_{i}$ in the next mission

Effective age of element $i$ at the beginning of the $k$ th mission

Effective age of element $i$ at the end of the $k$ th mission

Binary variable representing the status of element $i$ at the beginning of the $k$ th mission

Binary variable representing the status of element $i$ at the end of the $k$ th mission

Duration of the $k$ th mission

Fixed maintenance cost for element $i$

Corrective repair cost for replacement of the failed element $i$

Preventive repair cost for replacement of the functioning element $i$

Corrective/preventive repair cost allocated for the failed/functioning element $i$ after the $k$ th mission Total maintenance cost allocated for element $i$ after the $k$ th mission

Age reduction factor of element $i$ resulting from the maintenance subsequent to the $k$ th mission Scale parameter of the Weibull distribution of element $i$ 


$\begin{array}{ll}\beta_{i} & \begin{array}{l}\text { Shape parameter of the Weibull distribution of } \\ \text { element } i\end{array} \\ m_{i}^{f} & \begin{array}{l}\text { Characteristic constant associated with the } \\ \text { corrective repair cost of element } i\end{array} \\ m_{i}^{p} & \begin{array}{l}\text { Characteristic constant associated with the } \\ \text { preventive repair cost of element } i\end{array} \\ r_{i}(k) & \begin{array}{l}\text { Probability of element } i \text { surviving at the end of } \\ \text { the } k \text { th mission }\end{array} \\ R(k, w) & \begin{array}{l}\text { Probability of the entire MSS completing the } k \text { th } \\ \text { mission under demand } w\end{array}\end{array}$

\section{INTRODUCTION}

M AINTENANCE is crucial to system performance and reliability in industry. Maintenance strategy is a hot issue, with the aim at finding trade-offs between maintenance expenditure and system risk or/and profits [1]. In many industrial or military environments, it is very common that systems are required to perform a sequence of missions with finite breaks between any two consecutive missions. However, it is often impossible to perform all the desirable maintenance actions due to the limitations on maintenance resources, such as maintenance budget, duration of maintenance time within each break, and limited repairmen. In such cases, the decision-maker needs to identify a subset of activities among the set of feasible maintenance actions, and this strategy is called selective maintenance [2].

Rice et al. [3] first develop a mathematical programming model to optimize the selective maintenance problem for a parallel-series system with identical components. Cassady et al. [2] state that selective maintenance is widely used in industry as maintenance resources are limited. They establish a general framework for selective maintenance with binary state components by removing the structural restriction on subsystems, and discuss the optimization models for the bridge system, and complex systems with series, and parallel structures through general optimization models. To make the problem more practical, Cassady et al. [4] consider the case in which components' lifetimes follow a Weibull distribution, and multiple maintenance actions can be selected, such as minimal repair, and corrective or preventive replacement. A mathematical programming model is adopted to solve the optimization problem. Schneider \& Cassady [5] deal with the selective maintenance problem for a fleet aiming to maximize the probability that the fleet successfully completes the missions under limited maintenance resources. To improve efficiency of the optimization process, Rajagopalan \& Cassady [6] propose four improved enumerative procedures that can reduce the CPU time. Lust et al. [7] emphasize that the selective maintenance problem for systems with a large number of components forms a complex combinatorial problem, and the conventional enumeration method is no longer a feasible way. They develop three new methods: a construction heuristic, a heuristic based on the adaptation of Tabu search, and an exact method based on a branch and bound procedure, then apply them to various system configurations. More recently, Cassady et al. [8] incorporate features such as acquiring additional resources, establishing optimal constant resource capacities for the sequential missions, integrating redundancy allocations, and resource allocation in the system design phase. Schneider et al. [9] analyse a corrective selective maintenance model in a finite period with consecutive missions, and solve this multi-mission problem via a stochastic dynamic program. They conclude that the optimal selective maintenance policy for the single mission rarely differs from the multi-mission case.

Our literature survey indicates that the reported selective maintenance problems are mostly for binary state systems having only two states: working, or failed. Most real systems are multi-state systems (MSS), (see [10] for more examples of MSS in industrial applications). The selective maintenance for MSS has received an extensive amount of attention. Chen et al. [11] discuss selective maintenance optimization for MSS. They assume that a parallel-series system has $M$ subsystems, wherein each subsystem consists of $N_{i}$ identical components connected in parallel, and the component and system must be in one of the $K+1$ possible states. The model incorporated maintenance cost and transition probability matrices to describe the changes in the state.

In this paper, we study a selective maintenance policy for the MSS as defined in Lisnianski \& Levitin [10], [12], in which the states of the system are divided according to possible performance rates (levels). The MSS introduced here is distinct from the one in [11]. For example, a power generation system may have four states with respect to different power output capacities, say $0 \mathrm{MW}, 50 \mathrm{MW}, 80 \mathrm{MW}$, and $100 \mathrm{MW}$; and maintenance can only be performed within the early morning every Sunday. Because of the limited maintenance time interval, e.g. only four hours from 01:00 a.m. to 05:00 a.m., a subset among the set of feasible maintenance actions should be optimally selected under this limitation to guarantee the power output meets the user demand with maximum probability during the next week. To incorporate maintenance quality into the maintenance decision, an imperfect maintenance model (Kijima type II model) is used to give flexibility in terms of maintenance action options. A cost-maintenance quality relationship is constructed to reflect the imperfect maintenance quality as a function of maintenance cost, and it is more general than the approaches proposed in [4], [11]. With the involving of MSS, and the imperfect maintenance model, the simple non-linear 0-1 programming in the selective maintenance optimization for binary state systems changes into a complex non-linear continuous programming; and the enumerative methods proposed in the literature [2], [6] are inefficient, and time-consuming in this situation. A genetic algorithm (GA) is used to solve the resulting optimization problem.

The remainder of this paper is organized as follows. Section II gives a brief introduction to the concerned MSS in this paper, as well as some assumptions regarding the model formulation. Section III reviews the basic formula of the Kijima type II imperfect maintenance model, and the relationship linking maintenance quality to cost is investigated. The universal generating function method is introduced in Section IV to evaluate the probability of the repaired MSS successfully completing the next mission under random mission demand. The selective maintenance model is formulated in Section V. Section VI briefly introduces the genetic algorithm technique, and its representation and decoding process for the formulated optimization problem. An illustrative example of a coal transportation 
system, as well as some comparative studies, is presented in Section VII. Conclusions are followed in Section VIII.

\section{SYSTEM DESCRIPTION \& ASSUMPTIONS}

A system that can have a finite number of performance rates is called a MSS [10]. For example, if a flash memory chip in a computer system fails, the system can continue to operate, but with deteriorated memory capacity, and a power generating system has more than two possible states with different power output levels (e.g. $0 \mathrm{MW}, 50 \mathrm{MW}, 80 \mathrm{MW}$, etc.). There are various situations in which a system should be considered to be a MSS. Two examples include

1) a system consisting of different units that have a cumulative performance effect on the entire system, and

2) a system consisting of elements with variable performance due to deterioration (fatigue, partial failures, etc.) and repair actions.

To analyse MSS behavior, one has to know the characteristics of its elements. Suppose the MSS consists of $M s$-independent elements. Any system element $i(i \in\{1, \ldots, M\})$ can have $n_{i}$ different states corresponding to its possible performance rates, which are represented by the set

$$
\mathbf{g}_{i}=\left\{g_{i, 1}, g_{i, 2}, \ldots, g_{i, n_{i}}\right\} .
$$

The performance rate $G_{i}(t)$ of element $i$ at any instant $t(\geq 0)$ is a random variable which takes its values from $\mathrm{g}_{i}: G_{i}(t) \in \mathrm{g}_{i}$. Therefore, over the time interval $[0, T]$, the performance rate of the element $i$ needs to be modeled as a stochastic process. The probability distribution associated with different states of the MSS at any instant $t$ can be represented by the set

$$
\mathbf{p}_{i}(t)=\left\{p_{i, 1}(t), p_{i, 2}(t), \ldots, p_{i, n_{i}}(t)\right\},
$$

where $p_{i, j}(t)\left(1 \leq j \leq n_{i}\right)$ represents the probability that $G_{i}(t)=g_{i, j}$. The state probabilities satisfy the condition $\sum_{j=1}^{n_{i}} p_{i, j}(t)=1$, because at any instant $t$, the element can always be in one and only in one of $n_{i}$ states, and all the states of the element compose the complete group of mutually exclusive events.

The entire MSS may have $N_{s}$ different states corresponding to the cumulative performance rates, which is unambiguously determined by the system configuration, and performance rates of elements. Possible performance rates are represented by the set $\mathbf{G}_{s}=\left\{g_{1}, \ldots, g_{N_{s}}\right\}$. Thus, the MSS performance rate at any time instant $t$ is also a random variable $G(t)$ which takes values from the set $\mathbf{G}_{s}: G(t) \in \mathbf{G}_{s}$, and is determined by

$$
G(t)=\phi\left(G_{1}(t), \ldots, G_{M}(t)\right) .
$$

The instantaneous probabilities associated with the individual system state can be denoted by the set

$$
\mathbf{p}(t)=\left\{p_{1}(t), p_{2}(t), \ldots, p_{N_{s}}(t)\right\},
$$

where $p_{j}(t)$ represents the probability that $G(t)=g_{j}$.

The MSS considered in this work is defined with some basic assumptions as follows.

1) The MSS consists of $M$ binary states elements, and performance rates for each element $i(i \in\{1, \ldots, M\})$ are denoted by the set $\mathbf{g}_{i}=\left\{g_{i, 1}, g_{i, 2}\right\}$, where $g_{i, 2}(\neq 0)$ is a nominal performance rate, and $g_{i, 1}=0$ represents failure.

2) The MSS can be constructed by elements in arbitrary configuration, such as series-parallel, bridge, complex network, etc.

3) At the beginning of the $k$ th mission, the status of element $i$ is represented by binary variable $X_{i}(k)$, where

$$
X_{i}(k)= \begin{cases}1 & \text { if the element } \mathrm{i} \text { is functioning } \\ 0 & \text { if the element } \mathrm{i} \text { is in failure state. }\end{cases}
$$

After the $k$ th mission is over, the status of element $i$ is given by a binary variable $Y_{i}(k)$, where

$$
Y_{i}(k)= \begin{cases}1 & \text { if the element } \mathrm{i} \text { is functioning } \\ 0 & \text { if the element } \mathrm{i} \text { is in failure state. }\end{cases}
$$

4) $A_{i}(k)$ represents the effective age of element $i$ at the beginning of the $k$ th mission, and $B_{i}(k)$ is the effective age of element $i$ when the $k$ th mission is over.

5) The duration of the $k$ th mission is denoted by $L(k)$.

6) Any maintenance action can only be executed during the break between two successive missions. In the break, there exists limited resource (e.g. cost, time, repairmen, etc.) to perform maintenance. Decision-makers need to determine how to allocate the maintenance resource to individual element with the aim at restoring the entire MSS to the state which maximizes the probability of successfully completing the subsequent mission.

7) The probability of the system successfully completing a mission is defined as the probability that the performance rate of the MSS is not less than the demand level during the whole mission.

8) Multiple maintenance actions can be chosen for both failed, and functioning elements, including minimal repair, corrective/preventive replacement (or perfect maintenance), and imperfect maintenance. The maintenance quality relates with maintenance cost (or resources), and their relationship can be measured statistically.

The problem, and assumptions presented above are realistic in industrial fields [4]. Applications includes production and power generation systems with weekly or monthly maintenance, transportation and vehicle systems maintained between jobs, and military systems recovered only between missions. In these situations, systems have multiple performance rates due to the degradation of elements, and the maintenance resources might be under budget, or the maintenance time between consecutive missions might be limited.

\section{IMPERFECT MAINTENANCE QUALITY \& RELATED Cost}

In the literature related to maintenance strategy, a system is typically assumed to be restored to a condition either as good as new, or as bad as old prior to failure. This assumption is not realistic as discussed in [13]. It is more reasonable to assume that maintenance restores a system to a condition somewhere between these two extreme states. This maintenance is referred to as imperfect maintenance. A multitude of literature investigate modeling imperfect maintenance in various fashions, and the most relevant efforts among them are the $(p, q)$ model (see Nakagawa [14], [15]), the $(p(t), q(t))$ model (see Block et al. [16], 


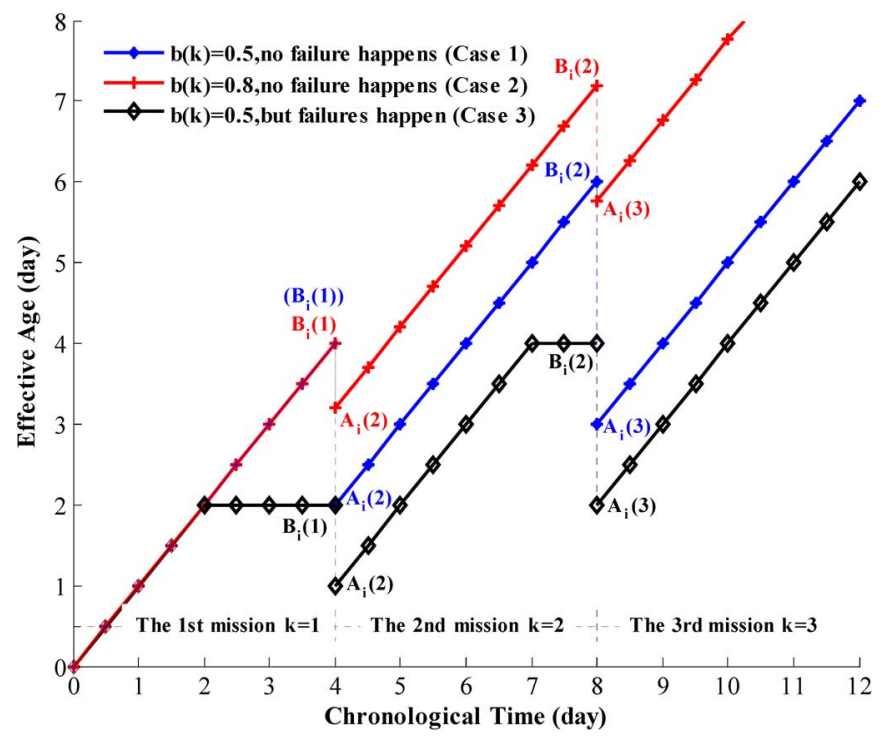

Fig. 1. Chronological time versus effective age in the Kijima type II model.

[17]), the $(p(n, t), q(n, t), s(n, t))$ model (see Makis \& Jardine [18]), the Kijima Type I and II models (see Kijima et al. [19], [20]), the improvement factor method (see Malik [21]), the hybrid imperfect model (see Lin et al. [21]), the geometric process (see Lam [23], [24]), and the quasi-renewal model (see Wang \& Pham [25]). Some applications of these imperfect maintenance models can be found in [26]-[29]. Nevertheless, the literature on imperfect maintenance deals with system models mainly based on binary state characteristics [13]. In MSS context, Levitin et al. [30], and Nahas et al. [31] apply the age reduction model to MSS with binary performance rate elements, and the optimal maintenance is determined for a finite horizon of time. The $\mathrm{Ki}$ jima type II model (see [19], [20]) is an appropriate way to describe imperfect maintenance for binary state elements, and it is employed in this work as a specified imperfect maintenance model to reflect the improvement caused by maintenance activity via the effective age of the element.

In accordance with the Kijima type II age reduction model, the effective age of any element $i$ after the maintenance subsequent to the $k$ th mission is given by

$$
A_{i}(k+1)=b_{i}(k) B_{i}(k) .
$$

If the binary state element $i$ fails during the $k$ th mission, its effective age will immediately stop increasing with chronological time as shown in Case 3 in Fig. 1. In this figure, failures happen, respectively, at the 2nd, and 7th days of chronological time; and the effective age of the element is steady at the remaining mission time. $b_{i}(k)\left(0 \leq b_{i}(k) \leq 1\right)$ is the age reduction factor representing maintenance quality, and a smaller $b_{i}(k)$ means a greater improvement as plotted in Fig. 1 (see Cases 1, and 2 where no failure happens). After finishing the $k$ th mission, elements may be either failed, or functioning. For the failed element, if one sets $b_{i}(k)=1$, it means there is no age reduction after the maintenance action, so that case corresponds to a condition as bad as old, or minimal repair. And for the functioning element, $b_{i}(k)=1$ means to do nothing. When $b_{i}(k)=0$, the element becomes as good as new after maintenance; and it can be considered as a corrective, and preventive replacement for failed, and functioning elements, respectively.

Based on the effective age model, the conditional survival probability after a maintenance activity is given by

$$
\begin{aligned}
R(x) & =1-\operatorname{Pr}\{X-t \leq x \mid X>t\} \\
& =1-\frac{\operatorname{Pr}\{t<X \leq x+t\}}{\operatorname{Pr}\{X>t\}} \\
& =\frac{\operatorname{Pr}\{X>x+t\}}{\operatorname{Pr}\{X>t\}}
\end{aligned}
$$

while the element is functioning at the beginning of mission with the effective age equal to $t$. When the failure distribution of the element $i$ follows the Weibull distribution while the element is functioning at the beginning of the $k$ th mission with cumulative effective age $A_{i}(k)$, the probability of element $i$ surviving at the end of the $k$ th mission is written as

$$
r_{i}(k)=\exp \left[-\left(\frac{L(k)+A_{i}(k)}{\eta_{i}}\right)^{\beta_{i}}+\left(\frac{A_{i}(k)}{\eta_{i}}\right)^{\beta_{i}}\right],
$$

In general, the more cost allocated for maintenance, the better the maintenance quality. As mentioned in [32], the spent maintenance cost, and the age of the system are the two major influences on the improvement factor which is regarded as $b_{i}(k)$ for each element in the present paper. If the spent maintenance cost approaches zero, the improvement approaches zero; thus, the repaired element would be in a condition as bad as old prior to failure. Oppositely, if the maintenance cost approaches the cost for replacement, the improvement would approach as good as new. On the other hand, the system age is another important factor which influences the improvement caused by maintenance. A great improvement may cost a little when the system is young. However, as a system ages, the improvement may get smaller, even at a large maintenance cost. Malik [21] proposes to determine the maintenance improvement factor through expert judgment. Lie \& Chun [32] implement a set of formulae to represent the accurate relationship between the ratios of preventive maintenance cost to preventive replacement cost, and the system improvement factor.

In this paper, we model this maintenance cost for any element $i$ after the $k$ th mission as

$$
C_{i}(k)=c_{i}(k)+c_{i}^{0} .
$$

Let $c_{i}^{r f}$ denotes the corrective repair cost for replacement of failed element $i$. The age reduction factor as a function of the corrective repair cost is then defined as

$$
b_{i}(k)=1-\left(\frac{c_{i}(k)}{c_{i}^{r f}}\right)^{\frac{1}{m_{i}^{f}}},
$$

where $m_{i}^{f}\left(m_{i}^{f}>0\right)$ is a characteristic constant that determines the exact relationship between corrective repair cost and age reduction factor through (9). It is related to the inherent characteristic of the element and its age, and can be estimated via the collected repair cost, and reliability/failure data of the element. If $c_{i}(k)=0$, then $b_{i}(k)=1$, there is no reduction in age, and it corresponds to the case where a minimal repair is performed on 


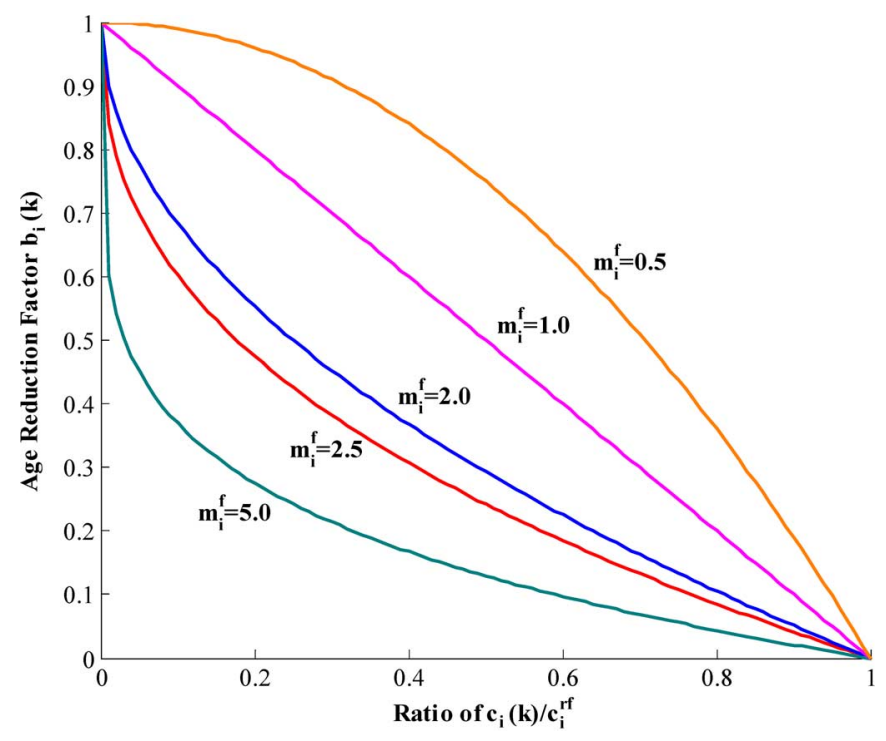

Fig. 2. The plot of $b_{i}(k)$ versus $c_{i}(k) / c_{i}^{r f}$ for different $m_{i}^{f}$.

the failed element $i$ between the break of the $k$ th mission and the $(k+1)$ th mission. Only the fixed maintenance $\operatorname{cost} c_{i}^{0}$ is involved to restore the element to a condition as bad as old prior to failure. Fig. 2 plots the trends of $b_{i}(k)$ versus $c_{i}(k) / c_{i}^{r f}$ for different $m_{i}^{f}$ values. It shows that the larger $m_{i}^{f}$ is, the more efficient it is when maintaining elements with a smaller amount of cost. The larger $m_{i}^{f}$ is corresponding to the element with younger age, because most items (or parts) are new in a "young age" element, and it is cheap and effective to repair a few failed parts of the element. For the aged element where every part is old, even renewing some parts is still ineffective at renewing the element's condition, therefore the associated $m_{i}^{f}$ should be smaller than that of younger elements. Thus, inherent characteristics of elements and its age are both important factors to determine the parameter $m_{i}^{f}$.

In the same manner, the age reduction factor as a function of the preventive repair cost is defined as

$$
b_{i}(k)=1-\left(\frac{c_{i}(k)}{c_{i}^{r p}}\right)^{\frac{1}{m_{i}^{p}}},
$$

where $m_{i}^{p}\left(m_{i}^{p}>0\right)$ is a characteristic constant that determines the exact relationship between preventive repair cost and the corresponding age reduction factor through (10). If $c_{i}(k)=0$, one has $b_{i}(k)=1$, and it denotes that no maintenance action is performed on the functioning element $i$. Therefore, the maintenance cost $C_{i}(k)$ is zero; otherwise, the total maintenance cost of element $i$ is the preventive repair cost allocated, plus the fixed maintenance cost.

Furthermore, with the assistance of the maximum likelihood estimation (MLE) regression method, as well as artificial neural networks (ANN), maintenance service data can be analysed to fit any other model, instead of (9) and (10), to set up the exact relationship between improvement and maintenance cost. Also, instead of cost, maintenance time can be used as a measure of maintenance quality because maintenance cost and time are related to each other.

\section{Probability of Successfully Completing a Mission}

The probability of successfully completing a mission is defined as the probability that the MSS performance rate is not less than the mission demand level during the whole single mission period. The universal generating function (UGF) method is employed to facilitate the probability assessment because it is a general, efficient method to evaluate the reliability of different types of MSS [10], [12].

The UGF represents the probability mass function of a discrete random variable via a polynomial form. In our case, the UGF can define the element or system state distribution, and all of the possible mutually exclusive states of the element or system can be represented via relating the probability of each state to the value that takes the random state variables corresponding to the element or system in that state. For any binary state element $i$, the performance rate distribution at any time instant can be given by

$$
u_{i}(z, t)=\sum_{j=1}^{2} p_{i, j}(t) \cdot z^{g_{i, j}} .
$$

And for a MSS, the performance rate distribution of the entire system at any time instant can be written as

$$
U_{s}(z, t)=\sum_{j=1}^{N_{s}} p_{j}(t) \cdot z^{g_{j}} .
$$

Therefore, to derive the UGF of an arbitrary structure system, one has to apply the composition operator $\otimes$ recursively as

$$
\begin{aligned}
U_{s}(z, t) & =\otimes\left\{u_{1}(z, t), \ldots, u_{M}(z, t)\right\} \\
& =\otimes\left\{\sum_{j_{1}=1}^{2} p_{1, j_{1}}(t) \cdot z^{g_{1, j_{1}}}, \ldots,\right. \\
\left.\sum_{j_{M}=1}^{2} p_{M, j_{M}}(t) \cdot z^{g_{M, j_{M}}}\right\} & \\
& =\sum_{j_{1}=1}^{2} \cdots \sum_{j_{M}=1}^{2}\left(\prod_{i=1}^{M} p_{i, j_{i}}(t) \cdot z^{\phi\left(g_{1, j_{1}}, \ldots, g_{M, j_{M}}\right)}\right) \\
& =\sum_{j=1}^{N_{s}} p_{j}(t) \cdot z^{g_{j}} .
\end{aligned}
$$

This polynomial $U_{s}(z, t)$ represents all of the possible mutually exclusive combinations of realizations of the variables by relating the probability of each combination to the value of function $\phi\left(G_{1}(t), \ldots, G_{M}(t)\right)$, which is determined by both the system structure, and performance rates combination property. For example, in the case of flow transmission type system with two elements connected in series, the composition function takes the form

$$
\phi\left(G_{1}(t), G_{2}(t)\right)=\min \left\{G_{1}(t), G_{2}(t)\right\} .
$$

And for the case where two elements are connected in parallel, the composition function is given by

$$
\phi\left(G_{1}(t), G_{2}(t)\right)=G_{1}(t)+G_{2}(t) .
$$


Because the MSS is degrading with time, if the performance rate of the MSS at the end of the next mission is not less than demand level, the mission is successfully completed. For functioning binary state element $i$ with the survival probability depending on the duration of the $k$ th mission, its UGF of the state distribution at the end of the $k$ th mission takes the form

$$
\begin{aligned}
u_{i}(z, L(k)) & =\sum_{j=1}^{2} p_{i, j}(L(k)) \cdot z^{g_{i, j}} \\
& =\left(1-r_{i}(k)\right) z^{g_{i, 1}}+r_{i}(k) z^{g_{i, 2}} .
\end{aligned}
$$

It denotes that element $i$ survives at the end of the $k$ th mission with probability $r_{i}(k)$, and fails with probability $1-r_{i}(k)$. If the element's lifetime follows the Weibull distribution, (7) can be directly substituted into (16). The state distribution of the MSS at the end of the $k$ th mission is derived via UGF as

$$
\begin{aligned}
U_{s}(z, L(k)) & =\otimes\left\{u_{1}(z, L(k)), \ldots, u_{M}(z, L(k))\right\} \\
= & \sum_{j_{1}=1}^{2} \cdots \sum_{j_{M}=1}^{2}\left(\prod_{i=1}^{M} p_{i, j_{i}}(L(k))\right. \\
\left.\cdot z^{\phi\left(g_{1, j_{1}}, \ldots, g_{M, j_{M}}\right)}\right) & \\
= & \sum_{j=1}^{N_{s}} p_{j}(L(k)) \cdot z^{g_{j}}
\end{aligned}
$$

where $p_{i, 1}(L(k))$, and $p_{i, 2}(L(k))$ are equal to the failure, and survival probabilities of element $i$, respectively (as shown in (16)). And $p_{j}(L(k))$ is the probability that the MSS is staying at state $j$ at the end of the $k$ th mission. Suppose the demand level of the $k$ th mission is $w$. The probability that the MSS completing the subsequent single mission (the $k$ th mission) can be written as

$$
R(k, w)=\sum_{i=1}^{N_{s}} p_{i}(L(k)) \cdot 1\left(F\left(g_{i}, w\right) \geq 0\right),
$$

where $1(x)$ is an indication function, and $F\left(g_{i}, w\right)=g_{i}-w$. If the demand level for the $k$ th mission is a random variable with $H$ possible values, the probability that the MSS successfully completes the $k$ th mission is given by

$$
R(k, w)=\sum_{i=1}^{H} q_{i} \sum_{j=1}^{N_{s}} p_{j}(L(k)) \cdot 1\left(F\left(g_{j}, w_{i}\right) \geq 0\right) .
$$

Due to budget, not all the failed elements will be restored to a functioning state before the $k$ th mission. Let the 0-1 binary vector $\mathbf{X}(k)=\left\{X_{1}(k), \ldots, X_{M}(k)\right\}$ represent the initial states of the elements at the beginning of the $k$ th mission. One combines the initial states into the UGF of elements to formulate the element's ability (in a probabilistic sense) to survival in the $k$ th mission. The UGF of element $i$ with considering the initial state at the beginning of the $k$ th mission is written as

$$
u_{i}\left(X_{i}(k), z, t\right)=X_{i}(k) \cdot u_{i}(z, t)+\left(1-X_{i}(k)\right) \cdot z^{0} .
$$

It is clear that $u_{i}\left(X_{i}(k), z, t\right)=u_{i}(z, t)$ for $X_{i}(k)=1$, representing that the element $i$ is in a functioning state before the $k$ th mission starts, and $u_{i}\left(X_{i}(k), z\right)=z^{0}$ for $X_{i}(k)=0$ represents no contribution to the system performance rate during the $k$ th mission. For instance, the combination operation for the UGF of two parallel-connected elements with incorporating their initial states can be written as

$$
\begin{aligned}
u_{1} & \left(X_{1}(k), z, t\right) \underset{\text { Par }}{\otimes} u_{2}\left(X_{2}(k), z, t\right) \\
= & {\left[X_{1}(k) u_{1}(z, t)+\left(1-X_{1}(k)\right) z^{0}\right] } \\
& \underset{\text { Par }}{\otimes}\left[X_{2}(k) u_{2}(z, t)+\left(1-X_{2}(k)\right) z^{0}\right] \\
= & {\left[X_{1}(k) X_{2}(k)\right] u_{1}(z, t) \underset{\text { Par }}{\otimes} u_{2}(z, t) } \\
& +\left[\left(1-X_{1}(k)\right) X_{2}(k)\right] z^{0} \underset{\text { Par }}{\otimes} u_{2}(z, t) \\
& +\left[X_{1}(k)\left(1-X_{2}(k)\right)\right] u_{1}(z, t) \\
& \underset{\text { Par }}{\otimes} z^{0}+\left[\left(1-X_{1}(k)\right)\left(1-X_{2}(k)\right)\right] z^{0} \underset{\text { Par }}{\otimes} z^{0} .
\end{aligned}
$$

Hence, the UGF of the MSS with the initial states of each element at the beginning of the $k$ th mission takes the form

$$
U_{s}(\mathbf{X}(k), z, t)=\otimes\left(u_{1}\left(X_{1}(k), z, t\right), \ldots, u_{M}\left(X_{M}(k), z, t\right)\right),
$$

and it can be solved via the composition operator mentioned above, recursively.

Because the selective maintenance for a MSS is executed before the next mission (the $k$ th mission), the system UGF at the end of the $k$ th mission can be recursively determined according to the initial state of each elements, and the UGF of the element state distribution at the end of the $k$ th mission. Therefore, the probability of the MSS successfully completing its next mission can be evaluated using (17) or (18).

\section{Modeling of Selective Maintenance}

\section{A. Maintenance Cost}

The total maintenance cost consists of two categories: PM cost for the selected functioning elements, and $\mathrm{CM}$ cost for the selected failed elements. Let binary decision variable $V_{i}(k)$ indicates whether PM is executed on functioning element $i$ or not after the $k$ th mission. If the functioning element $i$ is subject to PM action after the $k$ th mission, then $V_{i}(k)=1$; otherwise, $V_{i}(k)=0$. The total PM cost is

$$
C_{p}(k)=\sum_{i=1}^{M}\left[\left(c_{i}(k)+c_{i}^{0}\right) \cdot V_{i}(k) \cdot Y_{i}(k)\right],
$$

which denotes that element $i$ can be selected to perform PM only when the element is in a functioning state at the end of the $k$ th mission $\left(Y_{i}(k)=1\right)$, and the decision variable $V_{i}(k)$ is set to one. $c_{i}(k)$ represents the preventive repair cost allocated. If the failed element $i$ is subjected to corrective maintenance 
action, the binary decision variable $W_{i}(k)$ is set to one; otherwise, $W_{i}(k)=0$. The total cost of corrective maintenance cost is given by

$$
C_{r}(k)=\sum_{i=1}^{M}\left[\left(c_{i}(k)+c_{i}^{0}\right) \cdot W_{i}(k) \cdot\left(1-Y_{i}(k)\right)\right],
$$

where $\left(1-Y_{i}(k)\right)$ means the $\mathrm{CM}$ can only be executed on the failed element at the end of the $k$ th mission, and $c_{i}(k)$ represents the corrective repair cost allocated. Thus, the total maintenance cost for the MSS after the $k$ th mission is formulated as

$$
C(k)=C_{r}(k)+C_{p}(k) .
$$

\section{B. Selective Maintenance Modeling}

Given the state $Y_{i}(k)$, and effective age $B_{i}(k)$ of each element at the end of the $k$ th mission, the selective maintenance problem is twofold: identifying the element to be repaired (either preventive or corrective), where the associated binary decision variable are $V_{i}(k)$, and $W_{i}(k)$; and determining how much the maintenance cost should be allocated for each repair action, where the corresponding decimal decision variables are $c_{i}(k)$. Let the budget constraint on the total maintenance cost after the $k$ th mission be denoted by $C_{0}(k)$. The non-linear programming formulation to search the selective maintenance subset for maximizing the probability of successfully completing the $(k+1)$ th mission is developed as

$$
\begin{gathered}
\max R(k+1, w)=\sum_{i=1}^{H} q_{i} \sum_{j=1}^{N_{s}} p_{j}(L(k+1)) \\
\text { subject to } C(k) \leq C_{0}(k) \\
W_{i}(k)+V_{i}(k) \leq 1 \\
W_{i}(k)+Y_{i}(k) \leq 1 \\
\left.V_{i}(k)-Y_{i}(k) \leq 0\right) \\
A_{i}(k+1)=b_{i}(k) \cdot B_{i}(k) \\
X_{i}(k+1)=Y_{i}(k)+W_{i}(k) \\
X_{i}(k+1), Y_{i}(k), W_{i}(k), V_{i}(k) \text { binary }
\end{gathered}
$$

In the above formulations, the constraints given by (28)-(30) restrict that CM, and PM can only be selected to perform on failed, and functioning elements, respectively. Constraint (31) is the Kijima type II imperfect maintenance model, and the constraint given by (32) sets the state of the elements at the beginning of the $(k+1)$ th mission in accordance with its state at the end of the $k$ th mission, and the maintenance action taken. If maintenance time is used instead of cost, the constraint will be similar to that given by (27).

\section{GA Optimization TechniQue}

Selective maintenance optimization for a MSS with imperfect maintenance is a complex, non-linear, continuous programming problem as shown in (26)-(33). An exhaustive examination of all possible solutions is not realistic due to the compu-
TABLE I

The MAInTENAnCE ACtion, AND Cost CoRResponding To $s_{i}$ FOR FUNCTIONING ELEMENT $i$

\begin{tabular}{ccc}
\hline$s_{i}$ & Maintenance action & Maintenance cost $C_{i}(k)$ \\
\hline 0 & Do Nothing & 0 \\
$\vdots$ & $\vdots$ & $\vdots$ \\
$i$ & Imperfect PM & $c_{i}^{0}+i \cdot c_{i}^{r p} / N_{L}$ \\
$\vdots$ & $\vdots$ & $\vdots$ \\
$N_{L}$ & Preventive Replacement & $c_{i}^{0}+c_{i}^{r p}$ \\
\hline
\end{tabular}

tational time limitation. Meta-heuristic algorithms, such as genetic algorithm (GA), Tabu search, simulated annealing algorithm, and ant colony optimization (ACO), are efficient, effective approaches to search the global optimal solution (or approximate global optimal solution) of combinational, and non-linear programming problems. These algorithms do not require derivative information to determine the next direction of the search, and easily adapt well to other problems. Among these algorithms, genetic algorithm (GA) is one of the most widely used evolutionary methods. It is a popular, universal tool for solving various optimization problems because of its advantages, and has been successfully applied to an abundance of optimization problems in reliability engineering (see Levitin [33]) as well as maintenance optimization problems (see Levitin \& Lisnianski [30], [34]). Thus, GA is employed in this paper due to its flexibility in representing any kind of design variable, and good global optimization capability.

To apply the GA to a specific problem, solution representation is an important procedure which must be defined first. Because the amount of repair cost allocated to each element can be any real value among the feasible region, to reduce the computational intensity, decision variables are transformed into integral form, and the individual solution is represented by an integral string

$$
\mathbf{s}=\left\{s_{1}, s_{2}, \ldots, s_{M}\right\},
$$

where $s_{i}$ is a decimal digit representing the discrete maintenance quality level for element $i$, and $0 \leq s_{i} \leq N_{L}$, where $N_{L}$ denotes the maximum maintenance quality level determined by the decision-maker. For functioning, and failed elements respectively, the value of $s_{i}$ represents different maintenance action, and quality; and its corresponding costs are defined in Tables I and II. Based on the decoding solution, the binary variables in (26)-(33) are determined as, for functioning element $i$,

$$
\begin{cases}s_{i}=0, & \text { one has } Y(k)=1, X(k+1)=1, \\ & V_{i}(k)=0, W_{i}(k)=0 \\ s_{i}>0, & \text { one has } Y(k)=1, X(k+1)=1, \\ & V_{i}(k)=1, W_{i}(k)=0\end{cases}
$$

and for failed element $i$,

$$
\begin{cases}s_{i}=0, & \text { one has } Y(k)=0, X(k+1)=0, \\ & V_{i}(k)=0, W_{i}(k)=0 \\ s_{i}>0, & \text { one has } Y(k)=0, X(k+1)=1, \\ & V_{i}(k)=0, W_{i}(k)=1 .\end{cases}
$$

For example, consider a MSS consisting of four elements, with the states at the end of the $k$ th mission being \{failed, failed, 
TABLE II

The Maintenance Action, ANd Cost CoRRESPONDING to $s_{i}$ FOR FAILED ELEMENT $i$

\begin{tabular}{ccc}
\hline$s_{i}$ & Maintenance action & Maintenance cost $C_{i}(k)$ \\
\hline 0 & Do Nothing & 0 \\
1 & Minimal Repair & $c_{i}^{0}$ \\
$\vdots$ & $\vdots$ & $\vdots$ \\
$i$ & Imperfect CM & $c_{i}^{0}+(i-1) \cdot c_{i}^{r f} /\left(N_{L}-1\right)$ \\
$\vdots$ & $\vdots$ & $\vdots$ \\
$N_{L}$ & Corrective Replacement & $c_{i}^{0}+c_{i}^{r f}$ \\
\hline
\end{tabular}

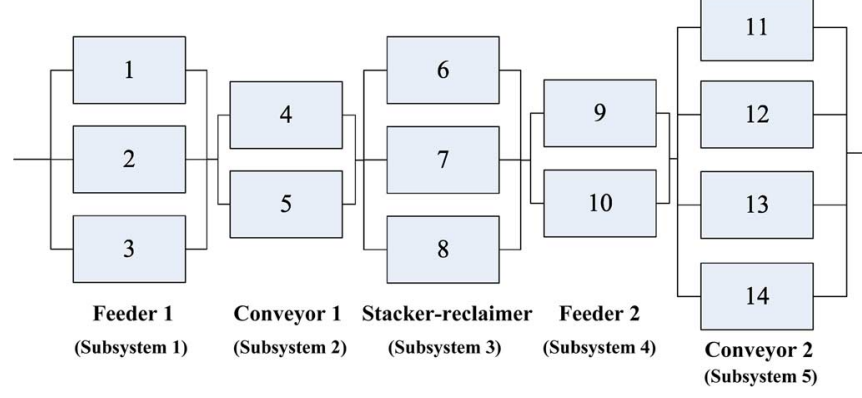

Fig. 3. Reliability block diagram of the coal transportation system.

good, good $\}$. The fixed maintenance cost for these elements is $\$ 5$. The corrective repair cost for replacement are respectively $\$ 30$, and $\$ 40$ for elements 1 , and 2; while the preventive repair cost for replacement are respectively $\$ 25$, and $\$ 20$ for elements 3, and 4. Let $N_{L}=10$. A specified individual solution $\mathbf{s}=\{1,6,0,10\}$ denotes that minimal repair is executed on element 1 with maintenance cost equal to $\$ 5$, and imperfect $\mathrm{CM}$ is performed on element 2 with maintenance cost equal to $\$ 5+(6-1) \times \$ 40 /(10-1)=\$ 27.22$. No action is executed on element 3 , and element 4 is preventively replaced by a new one with maintenance cost equal to $\$ 5+\$ 20=\$ 25$. The binary variables in (26)-(33) are

$$
\begin{aligned}
& Y_{1}(k)=0, X_{1}(k+1)=1, V_{1}(k)=0, W_{1}(k)=1, \\
& Y_{2}(k)=0, X_{2}(k+1)=1, V_{2}(k)=0, W_{2}(k)=1, \\
& Y_{3}(k)=1, X_{3}(k+1)=1, V_{3}(k)=0, W_{3}(k)=0, \text { and } \\
& Y_{4}(k)=1, X_{4}(k+1)=1, V_{4}(k)=1, W_{4}(k)=0 .
\end{aligned}
$$

The effective age at the beginning of the $(k+1)$ th mission is determined by (5) according to the effective age of the element at the end of the $k$ th mission. The age reduction factor relating to the repair cost allocated can be computed by (9), and (10).

\section{ILlustrative CASE}

The system is a coal transportation system in a power station that supplies a boiler, and consists of five basic subsystems as shown in Fig. 3. Feeder 1 loads the coal from the bin to conveyor 1. Conveyor 1 transports the coal to the stacker-reclaimer that lifts the coal up to the burner level. Feeder 2 loads conveyor 2 that supplies the burner feeding system of the boiler [35].

Each element is considered as a unit. Every subsystem consists of binary state elements. The values of parameters for each element, e.g. nominal performance rate (ton/hour), parameters
TABLE III

Parameters of Elements, Where Performance Rate is In TONS/Hour, TIME IS IN DAYS, AND COSTS ARE IN \$1,000 UNITS

\begin{tabular}{ccccccccccc}
\hline $\mathrm{ID}$ & $g_{i, 2}$ & $\eta_{i}$ & $\beta_{i}$ & $m_{i}^{p}$ & $c_{i}^{r p}$ & $m_{i}^{f}$ & $c_{i}^{r f}$ & $c_{i}^{0}$ & $B_{i}(k)$ & $Y_{i}(k)$ \\
\hline 1 & 55 & 1.5 & 25 & 2.5 & 15 & 2.5 & 25 & 3 & 35 & 1 \\
2 & 80 & 2.4 & 38 & 2.2 & 20 & 2.0 & 32 & 4 & 24 & 0 \\
3 & 120 & 1.6 & 28 & 2.6 & 25 & 3.0 & 35 & 3 & 45 & 0 \\
4 & 90 & 2.6 & 40 & 2.2 & 20 & 3.2 & 35 & 5 & 35 & 0 \\
5 & 145 & 1.8 & 28 & 1.8 & 25 & 4.0 & 34 & 2 & 28 & 1 \\
6 & 70 & 2.4 & 34 & 2.4 & 15 & 3.2 & 20 & 3 & 36 & 1 \\
7 & 95 & 2.5 & 26 & 2.8 & 24 & 3.0 & 30 & 6 & 44 & 0 \\
8 & 80 & 2.0 & 28 & 2.3 & 20 & 2.8 & 35 & 5 & 28 & 0 \\
9 & 95 & 1.2 & 26 & 2.0 & 18 & 2.5 & 28 & 3 & 38 & 1 \\
10 & 130 & 1.4 & 35 & 2.5 & 20 & 2.8 & 35 & 6 & 15 & 0 \\
11 & 50 & 2.8 & 40 & 3.2 & 22 & 3.0 & 32 & 7 & 30 & 0 \\
12 & 75 & 1.5 & 35 & 2.6 & 25 & 2.2 & 35 & 4 & 22 & 1 \\
13 & 85 & 2.4 & 30 & 2.8 & 18 & 2.8 & 36 & 6 & 38 & 1 \\
14 & 95 & 2.2 & 45 & 2.2 & 15 & 2.6 & 38 & 3 & 35 & 0 \\
\hline
\end{tabular}

TABLE IV

MISSION DEMANDS

\begin{tabular}{cccccc}
\hline Demand (ton/hour) & 120 & 90 & 60 & 30 & 10 \\
\hline Probability & 0.1 & 0.25 & 0.35 & 0.2 & 0.1 \\
\hline
\end{tabular}

of the Weibull life distribution, maintenance cost, effective age, and status after last mission (the $k$ th mission), are tabulated in Table III. The units of time, and cost are days, and $\$ 1,000$, respectively. The uncertain demand for the $(k+1)$ th mission is distributed as shown in Table IV, with the required demand levels, and their corresponding probabilities. Suppose the duration of the $(k+1)$ th mission is $L(k+1)=10$ days. Although the system is still functioning at the end of the last mission (the $k$ th mission) without any maintenance action, the probability of it successfully completing the next mission (the $(k+1)$ th mission) is only 0.006 . Given the maintenance budget $C_{0}(k)=$ $\$ 200,000$ in the break after the $k$ th mission, one has to optimally allocate the maintenance cost to each element to maximize the probability of successfully completing the $(k+1)$ th mission.

As stated in previous sections, the selective maintenance optimization is no longer a simple non-linear 0-1 programming problem. The GA method proposed in Section VI is used to search the global optimal solution with $N_{L}=7$, and the best maintenance strategy is presented as Scenario 1 in Table V. The optimal allocations of repair cost are listed in column "Cost" with the related fixed maintenance cost in parentheses.

From Table V (Scenario 1), one can see that all the elements are functioning at the start of the $(k+1)$ th mission, only elements 3 and 7 are subjected to corrective replacement, and element 5 is subjected to preventive replacement. All of the other failed elements are imperfectly repaired before the next mission is executed, and some functioning elements (elements 6 and 13) are subjected to imperfect PM. The probability of successfully completing the $(k+1)$ th mission is 0.77342 , and total maintenance cost is $\$ 199,880$. The optimal decision based on the method proposed in [4] which involves only minimal repair, preventive, and corrective replacement (Scenario 2) are also tabulated in Table $\mathrm{V}$. The probability of successfully completing the mission is 0.7336 , and corresponding total maintenance cost is $\$ 199,000$. As shown in Table V, although the maintenance cost 
TABLE V

OPTIMAL SOLUTIONS AND COMPARISON*

\begin{tabular}{|c|c|c|c|c|c|c|c|c|}
\hline \multirow{2}{*}{ Element ID } & \multicolumn{4}{|c|}{ With imperfect maintenance (Scenario 1) } & \multicolumn{4}{|c|}{ Without imperfect maintenance (Scenario 2) } \\
\hline & Action & Cost & $X_{i}(k+1)$ & $A_{i}(k+1)$ & Action & Cost & $X_{i}(k+1)$ & $A_{i}(k+1)$ \\
\hline 1 & - & 0 & 1 & 35 & PR & $15(3)$ & 1 & 0 \\
\hline 2 & IC & $5.33(4)$ & 1 & 14.2 & CR & $32(4)$ & 1 & 0 \\
\hline 3 & CR & $35(3)$ & 1 & 0 & $\mathrm{MC}$ & $0(3)$ & 1 & 45 \\
\hline 4 & IC & $17.5(5)$ & 1 & 6.82 & CR & $35(5)$ & 1 & 0 \\
\hline 5 & PR & $25.0(2)$ & 1 & 0 & - & 0 & 1 & 28 \\
\hline 6 & IP & $8.57(3)$ & 1 & 7.49 & - & 0 & 1 & 36 \\
\hline 7 & $\mathrm{CR}$ & $30(6)$ & 1 & 0 & $\mathrm{CR}$ & $30(6)$ & 1 & 0 \\
\hline 8 & IC & $5.84(5)$ & 1 & 13.23 & $\mathrm{MC}$ & $0(5)$ & 1 & 28 \\
\hline 9 & - & 0 & 1 & 38 & PR & $18(3)$ & 1 & 0 \\
\hline 10 & IC & $5.83(6)$ & 1 & 7.89 & $\mathrm{MC}$ & $0(6)$ & 1 & 15 \\
\hline 11 & IC & $5.34(7)$ & 1 & 13.49 & $\mathrm{MC}$ & $0(7)$ & 1 & 30 \\
\hline 12 & - & 0 & 1 & 22 & - & 0 & 1 & 22 \\
\hline 13 & IP & $5.14(6)$ & 1 & 13.71 & PR & $18(6)$ & 1 & 0 \\
\hline 14 & IC & $6.33(3)$ & 1 & 17.43 & $\mathrm{MC}$ & $0(3)$ & 1 & 35 \\
\hline$C(k+1)$ & \multicolumn{4}{|c|}{$\$ 199,880$} & \multicolumn{4}{|c|}{$\$ 199,000$} \\
\hline$R(k+1, w)$ & \multicolumn{4}{|c|}{0.77342} & \multicolumn{4}{|c|}{0.7336} \\
\hline
\end{tabular}

* The value in "Cost" column is the allocated repair cost with the fixed maintenance cost in parentheses, where costs are in $\$ 1,000$ units. Symbols denotation: “-”-Do Nothing; IC-Imperfect Corrective repair; CR-Corrective Replacement; IP-Imperfect Preventive repair; PR-Preventive Replacement; MC-Minimal Corrective repair.

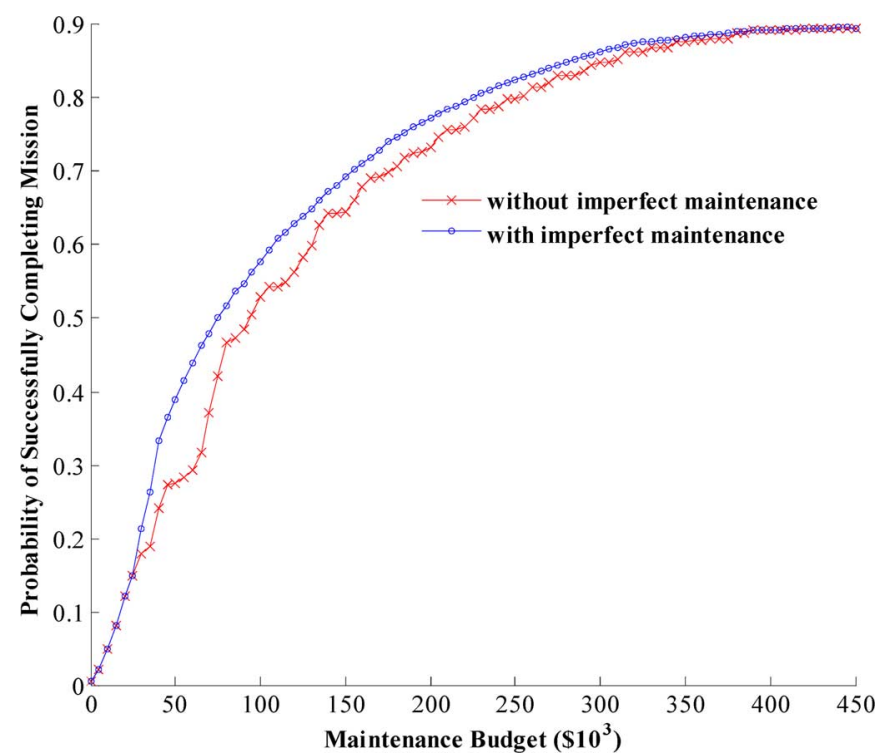

Fig. 4. Maximum probability of successfully completing mission versus cost constraint.

in Scenario 1 is slightly higher than Scenario 2 ( $\$ 880$ or $0.44 \%$ ), the probability of successfully completing the mission in Scenario 1 remarkably increases by nearly $5.43 \%$. If maintenance resources are unlimited, and either corrective or preventive replacement is performed on each element before the next mission (Scenario 3), the probability of mission success is 0.8947 with a total maintenance cost of $\$ 448,000$. It indicates the maintenance cost in Scenario 1 decreases by $55.38 \%$ as compared with Scenario 3 , while the probability of successfully completing the mission in Scenario 1 decreases by only $13.56 \%$.

Generally, the more maintenance cost is incurred, the higher the probability of successfully completing the mission will be achieved. The curves of budgets versus the probabilities of successfully completing the mission are plotted in Fig. 4. The curve with cross marks represents the maximum achievable probability obtained by enumerative methods without considering imperfect maintenance actions (Scenario 2) at each budget constraint, and the other curve with circular marks corresponds to Scenario 1, that imperfect maintenance actions are considered and solved through the proposed GA method. It is observed that, in the early phase (cost constraint is approximately less than $\$ 25,000$ ), these two scenarios have the same maximum probability of successfully completing the mission with identical maintenance actions, as tabulated in Tables VI and VII, where two cases with budgets equal to $\$ 10,000$, and $\$ 25,000$ are presented as illustrative examples. The improvement from considering imperfect maintenance (Scenario 1) is greater at the middle phase of the curves (budget is from $\$ 50,000$ to $\$ 200,000$ ), and approaches zero at the last phase (budget is more than $\$ 350,000$ ). The maximum improvement of mission success probability is 0.1453 when the budget is equal to $\$ 65,000$, and the average success probability improved from Scenario 2 to Scenario 1 is 0.0307 (or $4.57 \%$ on the average increasing ratio). Moreover, if only minimal repair is performed on all the failed elements, the sum of the cost is $\$ 39,000$ with corresponding mission success probability equal to 0.1963 , while the same cost can achieve higher mission success probability 0.33287 for Scenario 1 , and 0.2416 for Scenario 2. In the case where the budget constraint is equal to $\$ 50,000$, Tables VI and VII show that, to do minimal repair for failed element 4 and 7 while replacing element 6 is less effective than allocating more repair cost on both elements 4 and 6 (with approximately $41 \%$ improvement on mission success probability). Additionally, the curve for Scenario 1 is smoother than for Scenario 2, and approximating the curve with the regression technique becomes feasible. One may use a specified high-order polynomial function (or ANN, etc.) as a regression model to represent the trend that the probability varies with cost constraints. Based on the trend, the decision-maker might get more insights on determining the trade-off between the 
TABLE VI

Optimal Selective Maintenance Strategy at Different Cost Constraint For Scenario 1*

\begin{tabular}{ccccccccccccc}
\hline \multirow{2}{*}{$\begin{array}{c}\text { Element } \\
\text { ID }\end{array}$} & \multicolumn{3}{c}{$C_{0}=\$ 10,000$} & \multicolumn{3}{c}{$C_{0}=\$ 25,000$} & \multicolumn{3}{c}{$C_{0}=\$ 39,000$} & \multicolumn{3}{c}{$C_{0}=\$ 50,000$} \\
\cline { 2 - 12 } & Act. & Cost & $X_{i}$ & Act. & Cost & $X_{i}$ & Act. & Cost & $X_{i}$ & Act. & Cost & $X_{i}$ \\
\hline 1 & - & 0 & 1 & - & 0 & 1 & - & 0 & 1 & - & 0 & 1 \\
2 & MC & 4 & 1 & MC & 4 & 1 & MC & 4 & 1 & MC & 4 & 1 \\
3 & - & 0 & 0 & - & 0 & 0 & MC & 3 & 1 & MC & 3 & 1 \\
4 & MC & 5 & 1 & MC & 5 & 1 & IC & 8.9 & 1 & IC & 16.7 & 1 \\
5 & - & 0 & 1 & - & 0 & 1 & - & 0 & 1 & - & 0 & 1 \\
6 & - & 0 & 1 & - & 0 & 1 & IP & 9 & 1 & IP & 11.6 & 1 \\
7 & - & 0 & 0 & - & 0 & 0 & - & 0 & 0 & - & 0 & 1 \\
8 & - & 0 & 0 & MC & 5 & 1 & MC & 5 & 1 & MC & 5 & 1 \\
9 & - & 0 & 1 & - & 0 & 1 & - & 0 & 1 & - & 0 & 1 \\
10 & - & 0 & 0 & MC & 6 & 1 & MC & 6 & 1 & MC & 6 & 1 \\
11 & - & 0 & 0 & - & 0 & 0 & - & 0 & 0 & - & 0 & 0 \\
12 & - & 0 & 1 & - & 0 & 1 & - & 0 & 1 & - & 0 & 1 \\
13 & - & 0 & 1 & - & 0 & 1 & - & 0 & 1 & - & 0 & 1 \\
14 & - & 0 & 0 & MC & 3 & 1 & MC & 3 & 1 & MC & 3 & 1 \\
\hline$R(k+1, w)$ & & 0.049 & & & 0.1485 & & 0.33287 & & 0.38895 & \\
$C(k+1)$ & & $\$, 000$ & & $\$ 23,000$ & & $\$ 38,900$ & & & $\$ 49,300$ & \\
\hline
\end{tabular}

*The value in "Cost" column is the summation of the fixed maintenance cost and allocated repair cost, where costs are in $\$ 1,000$ units. Symbols denotation: “-”-Do Nothing; IC-Imperfect Corrective repair; CR-Corrective Replacement; IP-Imperfect Preventive repair; PR-Preventive Replacement; MC-Minimal Corrective repair. $X_{i}$ is short for $X_{i}(k+1)$.

TABLE VII

Optimal Selective Maintenance Strategy at Different Cost Constraint for Scenario 2*

\begin{tabular}{|c|c|c|c|c|c|c|c|c|c|c|c|c|}
\hline \multirow{2}{*}{$\begin{array}{l}\text { Element } \\
\text { ID }\end{array}$} & \multicolumn{3}{|c|}{$C_{0}=\$ 10,000$} & \multicolumn{3}{|c|}{$C_{0}=\$ 25,000$} & \multicolumn{3}{|c|}{$C_{0}=\$ 39,000$} & \multicolumn{3}{|c|}{$C_{0}=\$ 50,000$} \\
\hline & Act. & Cost & $X_{i}$ & Act. & Cost & $X_{i}$ & Act. & Cost & $X_{i}$ & Act. & Cost & $X_{i}$ \\
\hline 1 & - & 0 & 1 & - & 0 & 1 & - & 0 & 1 & - & 0 & 1 \\
\hline 2 & $\mathrm{MC}$ & 4 & 1 & $\mathrm{MC}$ & 4 & 1 & $\mathrm{MC}$ & 4 & 1 & $\mathrm{MC}$ & 4 & 1 \\
\hline 3 & - & 0 & 0 & - & 0 & 0 & $\mathrm{MC}$ & 3 & 1 & $\mathrm{MC}$ & 3 & 1 \\
\hline 4 & $\mathrm{MC}$ & 5 & 1 & $\mathrm{MC}$ & 5 & 1 & MC & 5 & 1 & $\mathrm{MC}$ & 5 & 1 \\
\hline 5 & - & 0 & 1 & - & 0 & 1 & - & 0 & 1 & - & 0 & 1 \\
\hline 6 & - & 0 & 1 & - & 0 & 1 & PR & 18 & 1 & PR & 18 & 1 \\
\hline 7 & - & 0 & 0 & - & 0 & 0 & - & 0 & 0 & $\mathrm{MC}$ & 6 & 1 \\
\hline 8 & - & 0 & 0 & $\mathrm{MC}$ & 5 & 1 & - & 0 & 0 & $\mathrm{MC}$ & 5 & 1 \\
\hline 9 & - & 0 & 1 & - & 0 & 1 & - & 0 & 1 & - & 0 & 1 \\
\hline 10 & - & 0 & 0 & $\mathrm{MC}$ & 6 & 1 & $\mathrm{MC}$ & 6 & 1 & $\mathrm{MC}$ & 6 & 1 \\
\hline 11 & - & 0 & 0 & - & 0 & 0 & - & 0 & 0 & - & 0 & 0 \\
\hline 12 & - & 0 & 1 & - & 0 & 1 & - & 0 & 1 & - & 0 & 1 \\
\hline 13 & - & 0 & 1 & - & 0 & 1 & - & 0 & 1 & - & 0 & 1 \\
\hline 14 & - & 0 & 0 & $\mathrm{MC}$ & 3 & 1 & $\mathrm{MC}$ & 3 & 1 & $\mathrm{MC}$ & 3 & 1 \\
\hline$R(k+1, w)$ & \multicolumn{3}{|c|}{0.049} & \multicolumn{3}{|c|}{0.1485} & \multicolumn{3}{|c|}{0.2416} & \multicolumn{3}{|c|}{0.2759} \\
\hline$C(k+1)$ & \multicolumn{3}{|c|}{$\$ 9,000$} & \multicolumn{3}{|c|}{$\$ 23,000$} & \multicolumn{3}{|c|}{$\$ 39,000$} & \multicolumn{3}{|c|}{$\$ 50,000$} \\
\hline
\end{tabular}

* The value in "Cost" column is the summation of the fixed maintenance cost and allocated repair cost, where costs are in $\$ 1,000$ units. Symbols denotation: “-”-Do Nothing; IC-Imperfect Corrective repair; CR-Corrective Replacement; IP-Imperfect Preventive repair; PR-Preventive Replacement; MC-Minimal Corrective repair. $X_{i}$ is short for $X_{i}(k+1)$.

budget constraint and the required probability of successfully completing subsequent mission.

To analyse the influence from the mission demand level, curves presenting the relationship between the probability of successfully completing the mission and the mission demand level, with a budget of $\$ 200,000$, are illustrated in Fig. 5. We plot the maximum probability corresponding to the demand level from 0 to 240 for Scenario 1 (the curve with circular marks), and 2 (the curve with cross marks). The probability definitely decreases while the demand increases, and the probability changes at the same point in these two scenarios (e.g. probabilities in these two scenarios both change at points where demand is equal to ..., 70, 75, 80, 85, 90, 95, 95, $120, \ldots)$. The mission demands at these points are equal to the states' performance rate of the entire MSS. However, the probability of successfully completing the mission in Scenario 1 is always higher than for Scenario 2. From these curves, the maximum improvement of the mission success probability is 0.0515 where mission demand is equal to 125 , and the average improvement of mission success probability from Scenario 2 to Scenario 1 is 0.0255 (or $5.51 \%$ on the average increase ratio). Furthermore, the improvement from Scenario 1 to Scenario 2 is slight when demand is less than 100 , and greater improvements occur when demand level is in ranges $100 \sim 140$, and $160 \sim$ 230. The mission demand level is an important factor affecting the improvement of considering imperfect maintenance actions in selective maintenance. In addition, if the case that the probabilities of Scenario 1 and 2 are approximately the same (like 


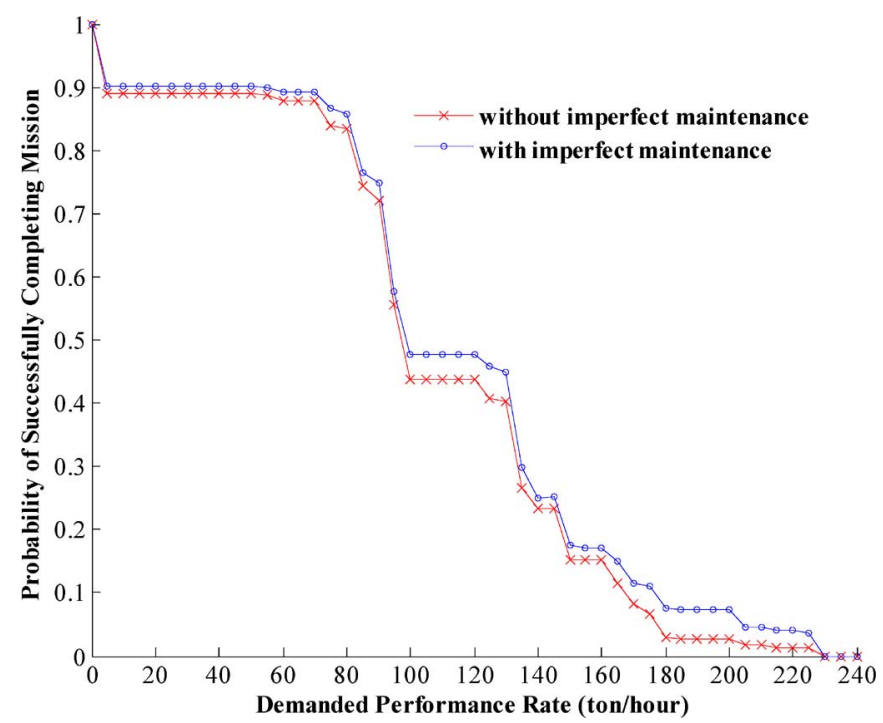

Fig. 5. Maximum probability of successfully completing mission versus demand level.

the situations that demand level is less than 100) happens, one may consider maintaining the MSS without considering the imperfect maintenance action. It is more convenient to maintain the unit with only minimal, and replacement actions.

\section{CONCLUSIONS}

In this paper, the selective maintenance problem in MSS context is addressed. Imperfect maintenance based on the Kijima type II model is used to formulate the improvement after maintenance, and a relationship linking maintenance quality with cost is proposed. Universal generating function is employed to facilitate the evaluation of the probability of successfully completing the next mission. Furthermore, the simple non-linear 0-1 programming in selective maintenance of binary state systems becomes a complex, non-linear continuous programming problem, which is impossible to solve through enumerative algorithms developed in earlier literature. GA is employed to solve the resulting optimization problem, and the comparisons are given between the proposed model, and the earlier method where imperfect maintenance quality is not accounted for. Incorporating imperfect maintenance action into selective maintenance yields better results.

Nevertheless, the limitation of this work is that every element is assumed to be a binary state element. To overcome this deficiency, the imperfect maintenance models for both multi-state elements, and MSS need to be further investigated. Meanwhile, it is worth considering the different consequences among the different kinds of imperfect maintenance models, such as Kijima type I and II models, for they own the different properties as mentioned in [36]. The trade-off between the mission success probability and budget, as well as other limited maintenance resources (e.g. maintenance time, repairmen, etc) needs addressing through multi-objective optimization approaches. Additionally, simulating the dynamic probability of successfully completing missions with considering the optimal maintenance resource allocation for multiple subsequent missions will be explored in our future work.

\section{ACKNOWLEDGMENT}

The authors would like to thank the associate editor, manager editor, and reviewers for their comments for improving this paper.

\section{REFERENCES}

[1] H. Z. Wang, "A survey of maintenance policies of deteriorating systems," European Journal of Operational Research, vol. 39, no. 3, pp. 469-489, 2002.

[2] C. R. Cassady, E. A. Pohl, and W. P. M. , Jr, "Selective maintenance modeling for industrial system," Journal of Quality in Maintenance Engineering, vol. 7, no. 2, pp. 104-117, 2001.

[3] W. F. Rice, C. R. Cassady, and J. A. Nachlas, "Optimal maintenance plans under limited maintenance time," in Proceedings of the Seventh Industrial Engineering Research Conference, 1998.

[4] C. R. Cassady, W. P. M., Jr, and E. A. Pohl, "Selective maintenance for support equipment involving multiple maintenance actions," European Journal of Operational Research, vol. 129, no. 2, pp. 252-258, 2001.

[5] K. Schneider and C. R. Cassady, "Fleet performance under selective maintenance," in Proceedings Annual Reliability and Maintainability Symposium (RAMS), 2004, pp. 571-576.

[6] R. Rajagopalan and C. R. Cassady, "An improved selective maintenance solution approach," Journal of Quality in Maintenance Engineering, vol. 12, no. 2, pp. 172-185, 2006.

[7] T. Lust, O. Roux, and F. Riane, "Exact and heuristic methods for the selective maintenance problem," European Journal of Operational Research, vol. 197, no. 3, pp. 1166-1177, 2009.

[8] I. M. Iyoob, C. R. Cassady, and E. A. Pohl, "Establishing maintenance resource levels using selective maintenance," The Engineering Economist, vol. 51, no. 2, pp. 99-114, 2006.

[9] K. Schneider, L. M. Maillart, C. R. Cassady, and C. Rainwater, "Selective maintenance decision-making over extended planning horizons," IEEE Trans. Reliability, vol. 58, no. 3, pp. 462-469, 2009.

[10] A. Lisnianski and G. Levitin, Multi-State System Reliability Assessment, Optimization, Application. , Singapore: World Scientific, 2003.

[11] C. Chen, M. Q. H. Meng, and M. J. Zuo, "Selective maintenance optimization for multi-state systems," in IEEE Canadian Conference on Electrical and Computer Engineering, 1999, pp. 1477-1482.

[12] G. Levitin, The Universal Generating Function in Reliability Analysis and Optimization. , London: Springer, 2005.

[13] H. Pham and H. Z. Wang, "Imperfect maintenance," European Journal of Operational Research, vol. 93, no. 3, pp. 425-438, 1996.

[14] T. Nakagawa, "Optimum policies when preventive maintenance is imperfect," IEEE Trans. Reliability, vol. 28, no. 4, pp. 331-332, 1979.

[15] T. Nakagawa, "Imperfect preventive maintenance," IEEE Trans. Reliability, vol. 28, no. 5, p. 402, 1979.

[16] H. W. Block, W. S. Borges, and T. H. Savits, "Age dependent minimal repair," Journal of Application Probability, vol. 22, no. 2, pp. 370-385, 1985.

[17] H. W. Block, W. S. Borges, and T. H. Savits, "A general age replacement model with minimal repair," Naval Research Logistics, vol. 35, no. 5, pp. 365-372, 1988.

[18] V. Makis and A. K. S. Jardine, "Optimal replacement policy for a general model with imperfect repair," Journal of Operational Research Society, vol. 42, no. 2, pp. 111-120, 1992.

[19] M. Kijima, H. Morimura, and Y. Suzuki, "Periodical replacement problem without assuming minimal repair," European Journal of Operational Research, vol. 37, no. 2, pp. 194-203, 1988.

[20] M. Kijima, "Some results for repairable systems with general repair," Journal of Application Probability, vol. 26, no. 1, pp. 89-102, 1989.

[21] M. A. K. Malik, "Reliable preventive maintenance scheduling," AIIE Trans., vol. 11, no. 3, pp. 221-228, 1979.

[22] D. Lin, M. J. Zuo, and R. C. M. Yam, "General sequential imperfect preventive maintenance models," International Journal of Reliability, Quality \& Safety Engineering, vol. 7, no. 3, pp. 253-266, 2000.

[23] Y. Lam, "A note on the optimal replacement problem," Advances in Applied Probability, vol. 20, no. 2, pp. 479-482, 1988.

[24] Y. Lam, "Geometric processes and replacement problem," Acta Mathematicae Applicatae Sinica, vol. 4, no. 4, pp. 366-377, 1988.

[25] H. Z. Wang and H. Pham, Reliability and Optimal Maintenance. , London: Springer, 2006

[26] S. C. Yang and T. W. Lin, "On the application of quasi-renewal theory in optimization of imperfect maintenance policies," in Proceedings Annual Reliability and Maintainability Symposium (RAMS), 2005, pp. $410-415$. 
[27] Y. L. Zhang, R. C. M. Yam, and M. J. Zuo, "Optimal replacement policy for multistate repairable system," Journal of Operational Research Society, vol. 53, no. 3, pp. 336-341, 2002.

[28] Y. L. Zhang, R. C. M. Yam, and M. J. Zuo, "A bivariate optimal replacement policy for a multistate repairable system," Reliability Engineering \& System Safety, vol. 92, no. 4, pp. 535-542, 2007.

[29] G. J. Wang and Y. L. Zhang, "An optimal replacement policy for a twocomponent series system assuming geometric process repair," Computers \& Mathematics With Applications, vol. 54, no. 2, pp. 192-202, 2007.

[30] G. Levitin and A. Lisnianski, "Optimization of imperfect preventive maintenance for multi-state systems," Reliability Engineering \& System Safety, vol. 67, no. 2, pp. 193-203, 2000.

[31] N. Nahas, A. Khatab, D. Ait-Kadi, and M. Nourelfath, "Extended great deluge algorithm for the imperfect preventive maintenance optimization of multi-state systems," Reliability Engineering \& System Safety, vol. 93, no. 11, pp. 1658-1672, 2008.

[32] C. H. Lie and Y. H. Chun, "An algorithm for preventive maintenance policy," IEEE Trans. Reliability, vol. 35, no. 1, pp. 71-75, 1986.

[33] G. Levitin, "Genetic algorithms in reliability engineering," Reliability Engineering \& System Safety, vol. 91, no. 9, pp. 975-976, 2006.

[34] G. Levitin and A. Lisnianski, "Joint redundancy and maintenance optimization for multi-state series-parallel systems," Reliability Engineering \& System Safety, vol. 64, no. 1, pp. 33-42, 1999.

[35] M. Nourelfath and D. Ait-Kadi, "Optimization of series-parallel multistate systems under maintenance policies," Reliability Engineering \& System Safety, vol. 91, no. 9, pp. 975-976, 2006.

[36] M. Bartholomew-Biggs, M. J. Zuo, and X. Li, "Modeling and optimizing sequential imperfect preventive maintenance," Reliability Engineering \& System Safety, vol. 94, no. 1, pp. 53-62, 2009.
Yu Liu received the B.E., and M.E. degrees in mechatronics engineering from the University of Electronic Science and Technology of China. He is currently working towards a Ph.D. degree in the School of Mechanical, Electronic, and Industrial Engineering at University of Electronic Science and Technology of China, and is pursuing his Ph.D. research in the Department of Mechanical Engineering at Northwestern University, U.S.A., as a pre-doctoral visiting fellow. His research interests include design under uncertainty, reliability of multi-state systems, maintenance decisions, and optimization.

Hong-Zhong Huang (M'06) is a full professor, and the Dean of the School of Mechanical, Electronic, and Industrial Engineering at the University of Electronic Science and Technology of China, Chengdu, Sichuan, 611731, China He has held visiting appointments at several universities in Canada, USA, and elsewhere in Asia. He received a Ph.D. degree in Reliability Engineering from Shanghai Jiaotong University, China. He has published over 150 journal articles, and 5 books in the fields of reliability engineering, optimization design, fuzzy sets theory, and product development. He is a Regional Editor of the International Journal of Reliability and Applications; an Editorial Board Member for The International Journal of Reliability, Quality and Safety Engineering; International Journal of Quality, Statistics, and Reliability; International Journal of Reliability and Quality Performance; and International Journal of Performability Engineering. He received the William A. J. Golomski Award from the Institute of Industrial Engineers in 2006, and the Best Paper Award of The 8th International Conference on Frontiers of Design and Manufacturing in 2008. His current research interests include system reliability analysis, warranty, maintenance planning and optimization, and computational intelligence in product design. 